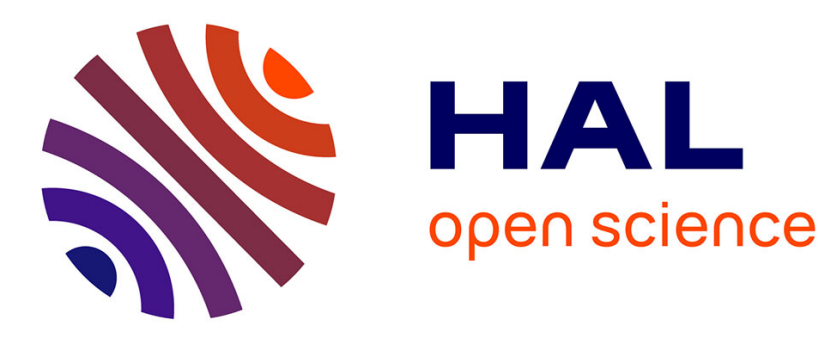

\title{
Label switching in mixtures
}

Christophe Biernacki, Vincent Vandewalle

\section{To cite this version:}

Christophe Biernacki, Vincent Vandewalle. Label switching in mixtures. Working Group on ModelBased Clustering Summer Session: Glasgow, July 17-23, 2011, Jul 2011, Glasgow, United Kingdom,

France. hal-03183299

\section{HAL Id: hal-03183299 \\ https://hal.inria.fr/hal-03183299}

Submitted on 27 Mar 2021

HAL is a multi-disciplinary open access archive for the deposit and dissemination of scientific research documents, whether they are published or not. The documents may come from teaching and research institutions in France or abroad, or from public or private research centers.
L'archive ouverte pluridisciplinaire $\mathbf{H A L}$, est destinée au dépôt et à la diffusion de documents scientifiques de niveau recherche, publiés ou non, émanant des établissements d'enseignement et de recherche français ou étrangers, des laboratoires publics ou privés. 


\section{Label switching in mixtures}

D, Université
Christophe Biernacki $i^{\mathrm{a}, \mathrm{c}}$, Vincent Vandewalle ${ }^{\mathrm{b}, \mathrm{c}}$ Laboratoire Paul Painlevé (Université Lille 1 - CNRS

${ }^{b}$ Équipe d'Accueil 2694 (Université Lille 2) ${ }^{c}$ Équipe MODAL (INRIA Lille Nord Europe)

\section{The label switching problem}

Mixture of $g$ distributions

$$
p(\cdot \mid \theta)=\sum_{k=1}^{g} \alpha_{k} p\left(\cdot \mid \beta_{k}\right)
$$

- $\alpha_{k}$ : mixtures weights $\left(\alpha_{k}>0\right.$ and $\left.\sum_{k} \alpha_{k}=1\right)$

- $\beta_{k}$ : parameters of each component distribution

- $\theta_{k}=\left(\alpha_{k}, \beta_{k}\right)$

\section{Generative interpretation}

$x=\left(x_{1}, \ldots, x_{n}\right)$ an $n$ i.i.d. sample from $p(\cdot \mid \theta)$

$z=\left(z_{1}, \ldots, z_{n}\right) \in$$$
\theta=\left(\theta_{1}, \ldots, \theta_{g}\right) \in \Theta
$$

Bayesian framework

- $p(\theta)$ a prior distribution on $\theta$

- Bayesian inference is based on the posterior distribution $p(\theta \mid x) \propto p(x \mid \theta) p(\theta)$

The problem

If $p(x \mid \theta)$ and $p(\theta)$ are invariant up to a mixture component renumbering then so does $p(\theta \mid x)$

- $\mathcal{P}_{g}$ is the set of $\{1, \ldots, g\}$ permutations

- $\sigma(\theta)=\left(\theta_{\sigma(1)}, \ldots, \theta_{\sigma(g)}\right)$ is the parameter $\theta$ permuted in index with $\sigma \in \mathcal{P}$

$$
p(\theta \mid x)=p(\sigma(\theta) \mid x)
$$

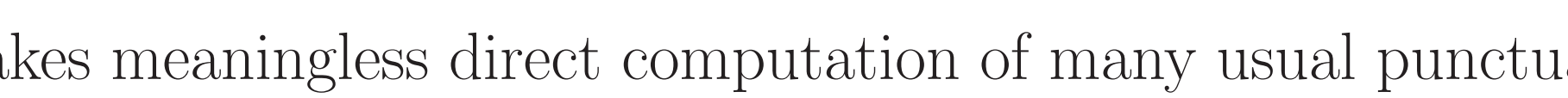
estimators as the posterior mean. The aim of many approaches is to remove this symmetiry.

\section{Illustration of the problem}

- Two univariate components $(g=2$

- $p\left(\cdot \mid \beta_{k}\right)=\mathcal{N}\left(\beta_{k}, 1\right)$

Proportions and variances known and fixed

- Mean $\theta_{1}=\beta_{1}$ et $\theta_{2}=\beta_{2}$ unknown

- Prior distributions on $\theta_{k} \sim \mathcal{N}(0,1)$ with $\theta_{1} \perp \theta_{2}$

- Posterior distributions

- $\theta_{k} \mid z, x \sim \mathcal{N}\left(n_{k} \bar{x}_{k} /\left(n_{k}+1\right), 1 /\left(n_{k}+1\right)\right)$

- $z_{i} \mid \theta, x \sim \mathcal{M}_{2}\left(1, t_{i 1}(\theta), t_{i 2}(\theta)\right)$

- $n_{k}=\sum_{i=1}^{n} \mathbb{I}_{z_{i}=k}$

$\bar{x}_{k}=\sum_{i=1} \mathbb{I}_{z_{i}=k} x_{i} / n_{k}$
$t_{i k}(\theta)=p\left(z_{i}=k \mid x, \theta\right)$
$-\theta_{1}=0$ and $\theta_{2}=0.25$

- $\theta_{1}=0$ and $\theta_{2}=0.25$

Two modes can be seen on the posterior distribution of $\theta_{1}$ when only one would be expected in absence of label
switching. It is then impossible to make relevant analysis of the posterior distributions component-wise.

\section{Standard solutions}

Modified prior distribution

- Artificial identifiability constraints on the parameters (Diebolt et Robert, 1994)

- Ordering constraints : $\theta_{1}<\theta_{2}$

- Modification of the prior distribution which becomes proportional to $p(\theta) \mathbb{I}_{\theta_{1}<\theta_{2}}$

- Not enough to solve the label switching problem (Celeux et al., 2000 ; Jasra et al., 2005)

$k$ - means algorithm on the parameters space

- Relabeling algorithm of the generated parameters (Stephens, 1997 ; Celeux, 1998)

- Find the permutation for the fixed parameter which minimizes a loss function

- $k$ - means type algorithm on the parameters space

- Underestimation of the dispersion of the posterior distribution

Invariant loss function

- Loss function invariant up to the parameters permutation (Celeux et al.. 2000)

- Choice of a loss function adapted to the inferential problem

- Optimization of this last

Probabilistic relabeling

- Probabilistic approach (Jasra et al., 2005) to take into account the uncertainness of the attribution of the permutation

to the parameters

Model on the deswitched posterior distribution learned from an unswitched sequence

- Probability for each permutation of the parameter get by the Gibbs sampler

- Computation of quantities of interest such as the posterior mean

Bibliographic overview

- Methods allowing to partially solve the problem

- Problem when posterior distributions are poorly separated, tuning parameters to set

- The latent partition is not taken into account

The latent partition is now used to solve the label-switching problem

\section{Bibliography}

1] Celeux, G., (1997) Discussion of 'On Bayesian analysis of mixtures models with an unknown number of components' with discussion), Journal of Royal Statistical Society: Series B, 59, 775-776.

2. Celeux, G., (1998) Bayesian inference for mixtures: the label-switching problem, R. Payne \&3 P. J. Greens, eds, CorstaT 98, Physica, Heidelberg, 227-232.

Celeux, G., Hurn, M. et Robert, C. P. (2000) Computational and Inferential Difficulties with Mixture Posterior

(4. Diebolt, J. et Robert, C. P. (1994) Estimation of finite mixture distributions, Journal of Royal Statistical Society

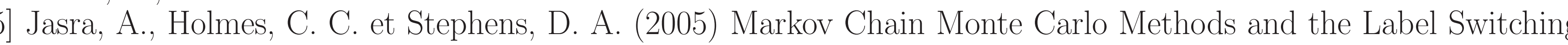
Shate Modeling, Statistical Science, 20, 1, 50-67.

Sperrin, M. and Jaki, T. and Wit, E. (2010) Probabilistic relabelling strategies for the label switching problem

7) Stephens, M. (1997) Bayesian Methods fo Mixtures of Normal Distribution, D. Phil. thesis, Department of Statistic niversity of Oxford.

\section{Idea: using the numbering information}

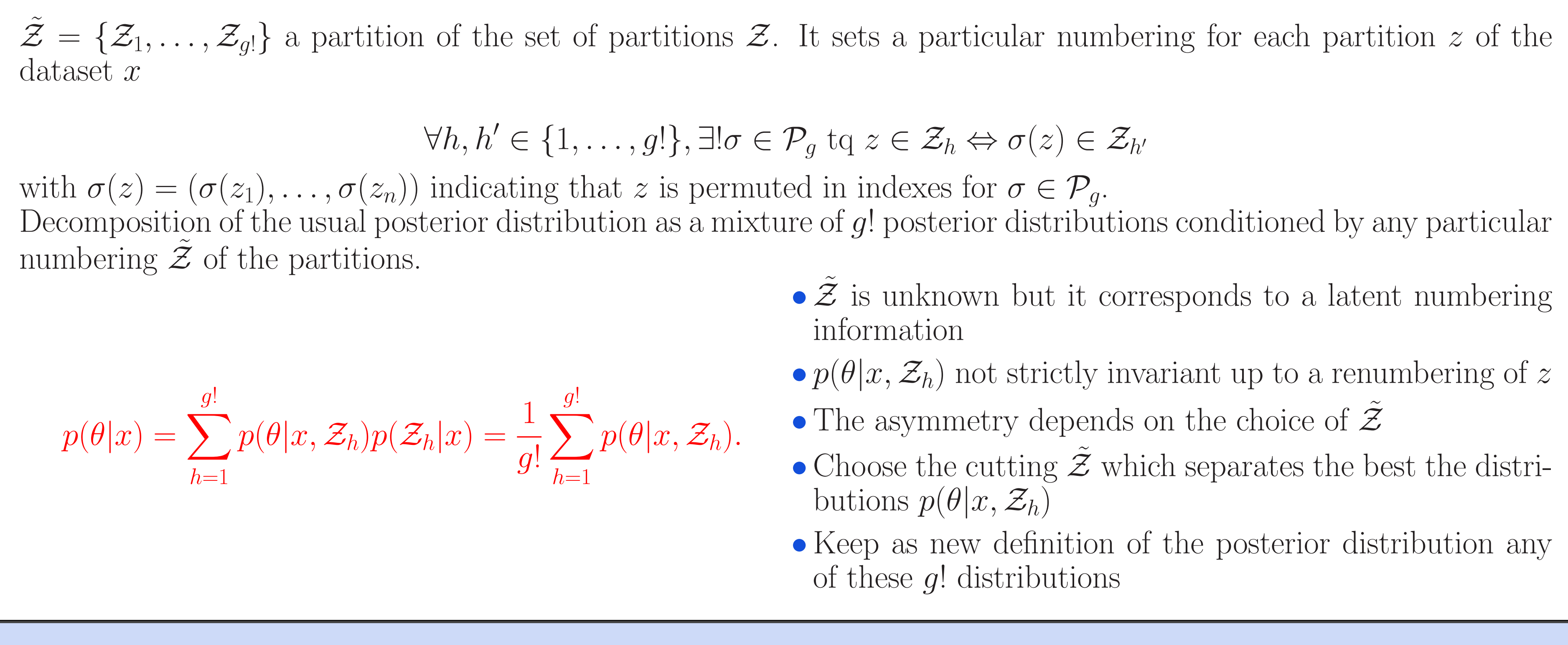

\section{Choosing a $g$ ! fraction $\tilde{\mathcal{Z}}$ of $\mathcal{Z}$}

Choice 1: $\tilde{\mathcal{Z}}^{K L}$

$\tilde{\mathcal{Z}}^{K L}$ maximizes the Kullback-Leibler divergence between the mixture components on $\mathcal{Z}_{h}$ which is written

$$
\tilde{\mathcal{Z}}^{K L}=\arg \max _{\mathcal{Z}} \min _{h=2, \ldots, g !} \int_{\Theta} p\left(\theta \mid x, \mathcal{Z}_{1}\right) \ln \left[\frac{p\left(\theta \mid x, \mathcal{Z}_{1}\right)}{p\left(\theta \mid x, \mathcal{Z}_{h}\right)}\right] d \theta .
$$

Choice 2: $\tilde{\mathcal{Z}}^{M A P}$

$$
\tilde{\mathcal{Z}}^{M A P}=\arg \max _{\mathcal{Z}} \min _{h=2, \ldots, g !} \frac{p\left(\theta^{M A P} \mid x, \mathcal{Z}_{1}\right)}{p\left(\theta^{M A P} \mid x, \mathcal{Z}_{h}\right)}
$$

It is equivalent to find the most probable numbering unit by unit computed in $\theta^{M A P}$ :

$$
\mathcal{Z}_{1}^{M A P}=\left\{z \in \mathcal{Z} / I d=\arg \max _{\sigma \in \mathcal{P}_{g}} p\left(\sigma(z) \mid x, \theta^{M A P}\right)\right\},
$$

$I d$ is the identity permutation. $\theta^{M A P}$ is the reference parameter for the numbering of the latent partition.

\section{Proposed Gibbs algorithm}

The classical Gibbs algorithm is slightly modified

$\bullet z \sim p(\cdot \mid x, \theta)$,

$z$ permuted in order to $\sigma(z) \in \mathcal{Z}_{1}{ }^{K L}$ or $\sigma(z) \in \mathcal{Z}_{1}{ }^{M A P}$

- $\theta \sim p(\cdot \mid x, \sigma(z))$

Additional algorithmic complexity negligible for $\mathcal{Z}_{1}{ }^{M A P}$

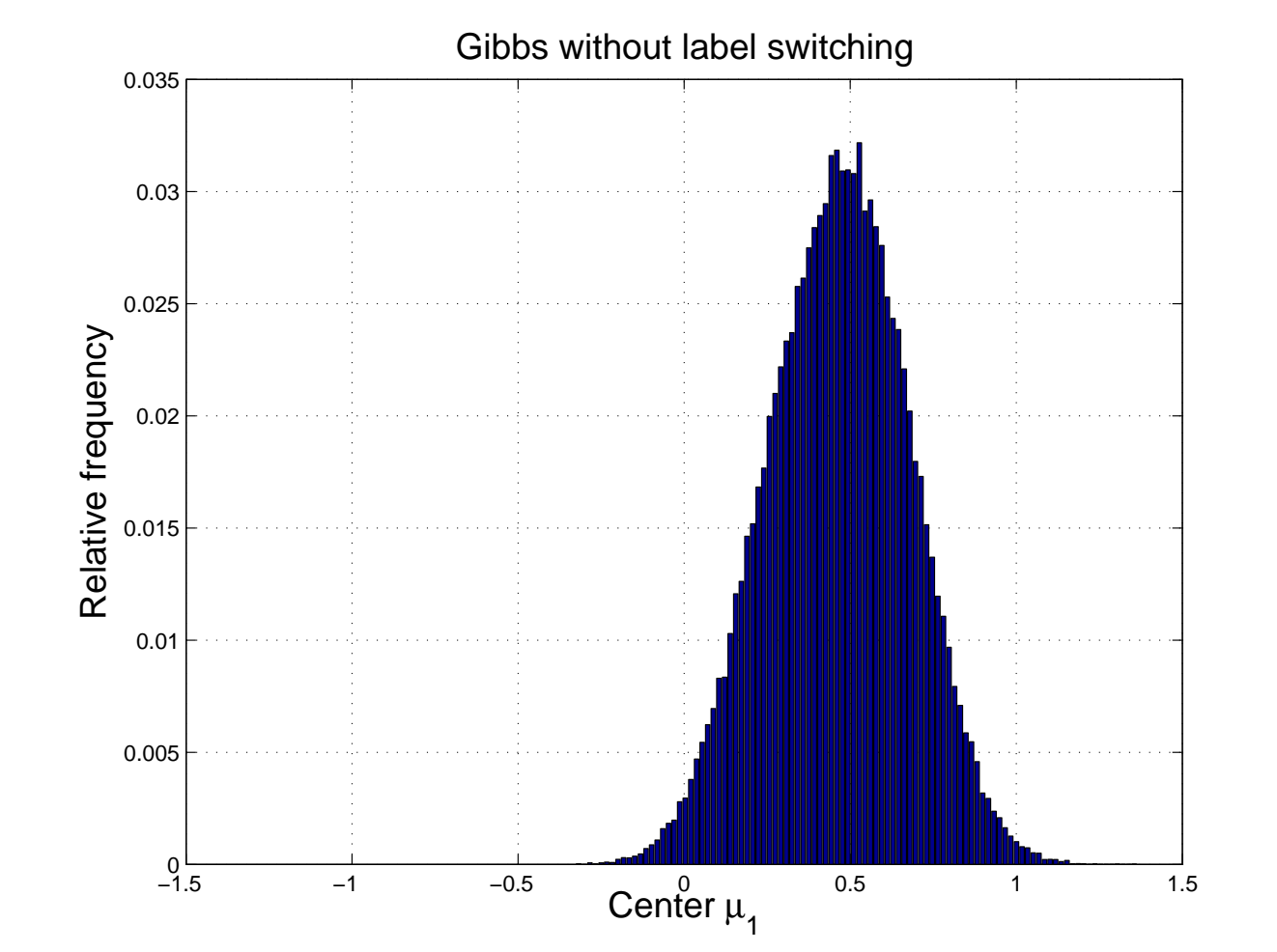

New posterior distribution $p\left(\theta_{1} \mid x, \mathcal{Z}_{1}^{M A P}\right)$

\section{Numerical experiments}

Experiments in the Gaussian setting (running example continued)

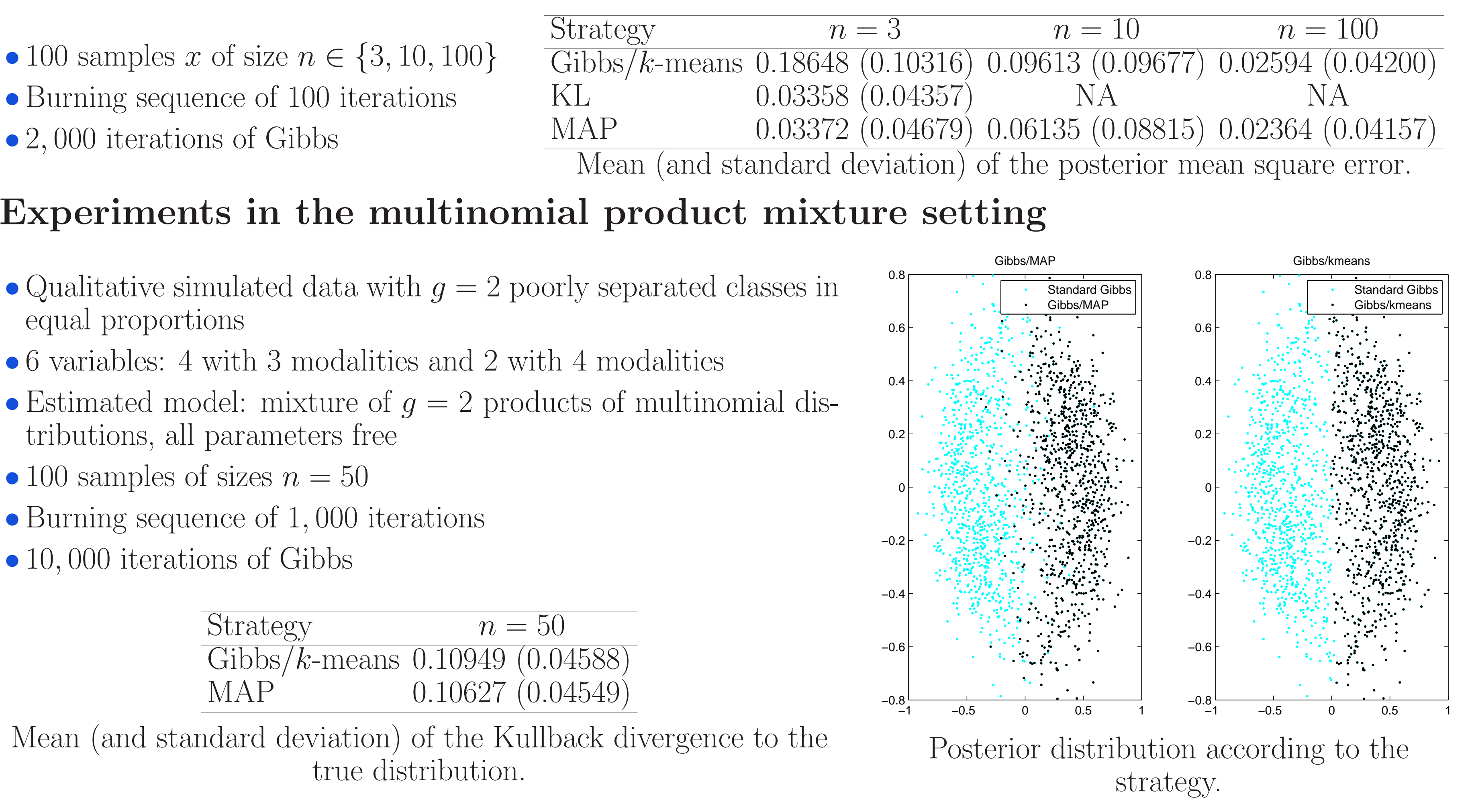

\section{Conclusion and perspectives}

Conclusion

- Separation of the posterior modes without break

- Assumption free on the unswitched distribution

- Computational cost similar to standard solutions

Perspectives

- Monitor the convergence of the Gibbs algorithm

- Many application areas: hidden Markov models, Potts model 